\title{
PRIMEROS ESTUDIOS BIOARQUEOLÓGICOS EN EL SITIO LOS TRES CERROS 1 (DEPARTAMENTO DE VICTORIA, ENTRE RÍOS)
}

\author{
Clara Scabuzzo ${ }^{1}$ y Agustina Ramos van Raap ${ }^{2}$ \\ ${ }^{1}$ CONICET-Dpto. Científico de Arqueología, F. Cs. Nat. y Museo, UNLP. clarascabuzzo@hotmail.com, \\ ${ }^{2}$ Dpto. Científico de Arqueología, F. Cs. Nat. y Museo, UNLP. magustina_rvr@hotmail.com
}

Presentado el: 30/10/2011 - Aceptado 01/12/2011

\section{Introducción}

El objetivo de este trabajo es dar a conocer los primeros resultados de las investigaciones bioarqueológicas llevadas a cabo en el sitio arqueológico Los Tres Cerros 1 (Delta Superior del río Paraná). Los temas que se presentan son: la conformación de la muestra (NMI, sexoedad), las modalidades de inhumación, el estado general de preservación de los esqueletos, los fechados radiocarbónicos y la descripción de algunas señales patológicas detectadas en los esqueletos. Estas investigaciones se encuadran dentro de un proyecto arqueológico más amplio dirigido por los doctores G. Politis y M. Bonomo cuyo objetivo es investigar la diversidad de los modos de vida y las trayectorias evolutivas de las poblaciones prehispánicas del Delta Superior del río Paraná.

El sitio Los Tres Cerros 1 (departamento de Victoria, Entre Ríos. Ver Figura 1) es un asentamiento de cazadores, recolectores, pescadores y horticultores a pequeña escala cuya subsistencia se centró en el consumo de animales acuáticos (peces, coipos y moluscos) y de vegetales domesticados (maíz, zapallo) (Bonomo et al. 2011; Politis et al. 2011). A su vez, las características de la tecnología cerámica sumada a la información cronológica relacionan estas ocupaciones con la entidad arqueológica redefinida por Ceruti (2003) como Goya-Malabrigo (Bonomo et al. 2011; Politis et al. 2011). Doce fechados radiocarbónicos disponibles para el sitio ubican las instalaciones humanas en el Holoceno tardío final entre 1030 y 560 años AP. El sitio bajo estudio corresponde a un montículo artificial, conocido como "cerrito". Estas elevaciones del terreno constituyeron puntos clave del paisaje donde los grupos llevaban a cabo actividades domésticas y rituales de entierro y cuya construcción demandó cierta organización del trabajo (Bonomo et al. 2011).

Dentro de la estructura del túmulo se recuperaron restos humanos, cuya distribución espacial permite conocer la presencia en el sitio de dos sectores bien diferenciados para la disposición de los muertos, un área concentrada de inhumaciones (núcleo) y un sector restringido. La mayoría de los individuos fueron hallados en lo que se determinó como el núcleo de inhumación. El mismo tiene alrededor de $4 \mathrm{~m}^{2}$ (cuadrículas 11 a 14) y estratigráficamente está ubicado en la base del cerrito. Las capas que contenían los esqueletos pre- 


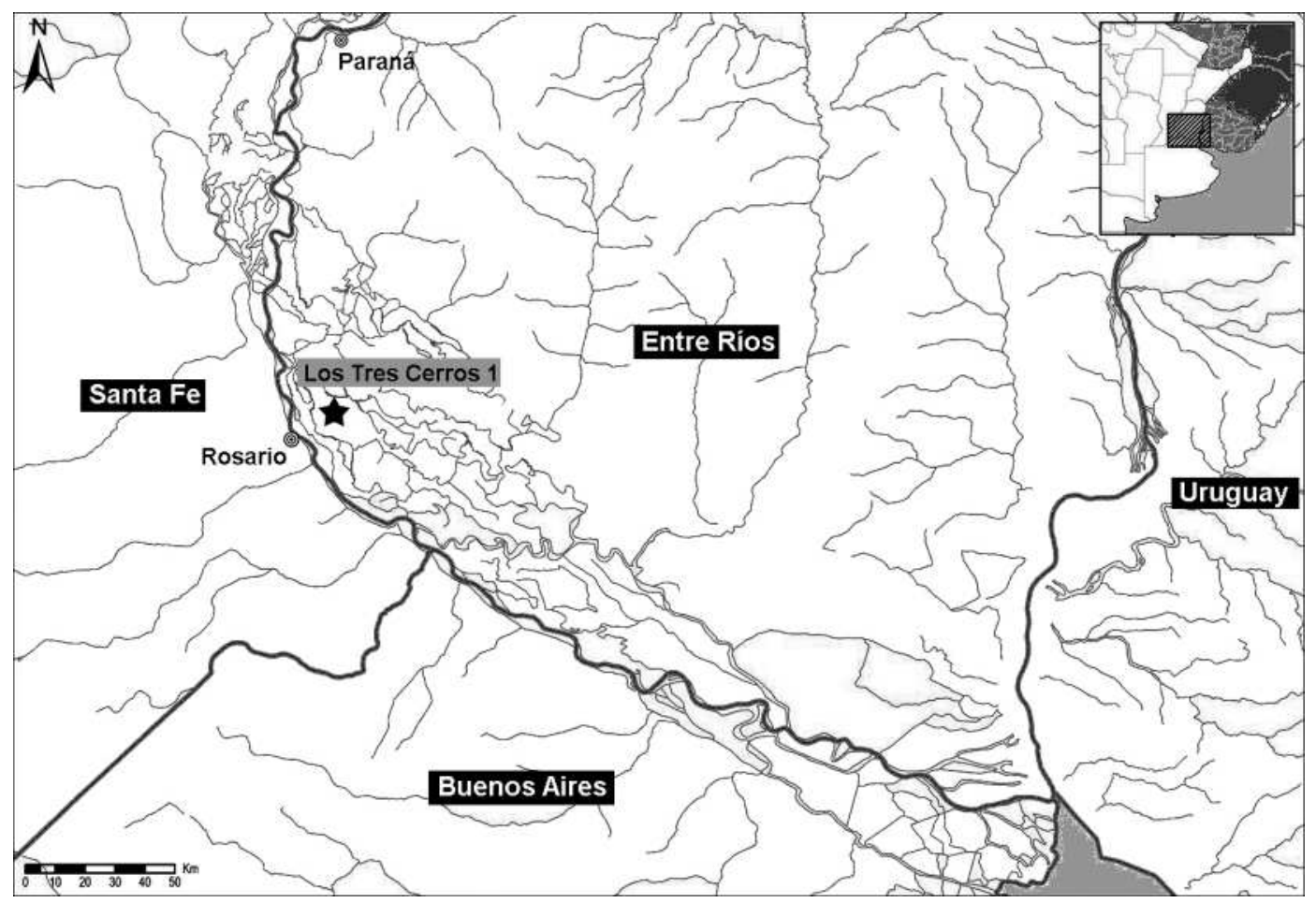

Figura 1. Ubicación del sitio Los Tres Cerros 1.

sentaban abundantes restos cerámicos y de alimentación, entre estos mandíbulas de coipos y vértebras de peces afectadas por el fuego, así como valvas de Diplodon sp.

El segundo sector se ubica estratigráficamente en la parte alta del cerrito, y lateralmente está alejado 10 metros del núcleo principal. Este es un sector discreto restringido a un fogón (cuadrícula 15) en cuyo interior se hallaron huesos humanos aislados asociados con restos de fauna y tiestos cerámicos.

Para los entierros se cuenta con dos fechados radiocarbónicos (650 \pm 70 y $775 \pm 85$ años AP) que ubican los eventos de inhumación en el Holoceno tardío final (Bonomo et al. 2011).

\section{Metodología}

El análisis bioarqueológico de los esqueletos comprendió dos etapas. La primera etapa estuvo orientada a la realización de un inventario general de los restos, a la determinación del número mínimo de individuos, al acondicionamiento de los elementos y al relevamiento de algunas variables tafonómicas (completitud de los elementos, presencia de depositaciones químicas y signos de termoalteración). Dentro de esta etapa también se realizaron las determinaciones de sexo y edad de los individuos, siguiendo los criterios bioarqueológicos estándar (Buikstra y Ubelaker 1994; White y Folkens 2005). Las estrategias metodológicas seguidas variaron según se tratara de individuos articulados o de restos aislados. Para las estimaciones de la edad se consideraron criterios cuantitativos y cualitativos. En los individuos subadultos se consideró: la fusión de epífisis de huesos largos (sensu Ribot y Roberts 1996), la erupción dentaria (Ubelaker 1999) y la longitud de los huesos largos. Para los individuos adultos los cambios en la sínfisis púbica, siguiendo a Todd (1921) y Brooks y 
Suchey (1990) (en White y Folkens 2005). Cada uno de los individuos fue asignado a una de las categorías etarias de Buikstra y Ubelaker (1994): feto, infante, niño, adolescente, adulto joven, adulto medio y adulto viejo. En el caso de las determinaciones de sexo se evaluaron las características de la pelvis y del cráneo y se realizaron medidas sobre huesos largos (ej. diámetro máximo de la cabeza del fémur). En el cráneo se observaron los arcos supraorbitarios, las crestas nucales, el tamaño de las apófisis mastoideas y la robustez mandibular. En la pelvis se tomó la profundidad y ancho de la escotadura ciática, concavidad subpúbica y la rama isquiopúbica (White y Folkens 2005).

La segunda etapa de trabajo, que aún está en desarrollo, tuvo como objetivo relevar la presencia de señales patológicas en los esqueletos. Se hicieron observaciones macroscópicas para determinar la presencia de patologías degenerativas a nivel de las articulaciones. Se relevaron todas las áreas de articulación y se clasificó el daño degenerativo en grados de severidad (Scabuzzo 2010). También se relevaron señales de reacciones periósticas, presencia de hiperostosis porótica o criba orbitalia en los cráneos y signos de traumatismos (Buikstar y Ubelaker 1994).

\section{Resultados}

Se analizaron 195 elementos de los cuales 145 (75\%) corresponden a individuos adultos, 43 (22\%) a individuos jóvenes-adolescente y 7 (3\%) a infantes de corta edad. En base al análisis de los huesos largos, sobre todo cúbito y radio, se determinó un número mínimo de ocho individuos inhumados en el sitio. De los cuales cuatro fueron identificados como adultos, tres como adultos jóvenes-adolescente y uno como un infante (perinato).

El análisis tafonómico mostró que los elementos en general se encontraban en un buen estado de conservación. El 54\% de los huesos tenía un grado de completitud 4, es decir que más del $75 \%$ del elemento estaba presente. El 20\% presentó completitud 3 y el $26 \%$ restante completitud 1 ó 2. En cuanto a la depositación química, 69 (36\%) elementos tenían óxido de manganeso sobre la superficie y en ningún caso se observó depositación de carbonato de calcio. El 8\% de los huesos presentaban signos de termoalteración. La mayoría de los elementos afectados aparecieron incluidos en el área discreta (fogón) dentro del sitio.

Finalmente, se observó la presencia de ocre sobre la superficie en 42 elementos (21\%). Este rasgo apareció asociado a individuos de distintas edades e inhumados tanto de manera primaria como secundaria y en los restos dispersos.

\section{Descripción de los entierros}

Dentro del área principal se recuperaron una inhumación secundaria múltiple, un entierro primario simple y huesos aislados (Individuos 1,2,3,4,5,6, y 7).

El paquete secundario múltiple está compuesto por los elementos óseos de al menos dos individuos. Para este entierro se cuenta con un fechado radiocarbónico de $650 \pm 70$ años AP (LP-2292). Uno de los esqueletos que componen el entierro secundario corresponde a una mujer adulta mayor (Individuo 1), representada por el cráneo fragmentado, las escápulas, una clavícula, ambos húmeros, un radio, ambos coxales, el sacro, los fémures, vértebras dorsales y lumbares, fragmentos de costillas, un carpo y un metatarso. En dos de los elementos -húmero y clavícula- se detectó pigmento rojo sobre la superficie. En varias articulaciones 
de este individuo se observó la presencia de osteoartritis, la misma afectó en los miembros superiores la cavidad glenoidea de la escápula izquierda, con desarrollo de osteofitos marginales más acentuados sobre el borde anterior de la cavidad. En los miembros inferiores en la articulación tibio-femoral se observó gran desarrollo osteofitico en la epífisis distal del fémur izquierdo y labiado en el derecho. El segmento corporal más dañado fue la columna, en la articulación del sacro con la quinta lumbar se detectó desarrollo de osteofitos mayores a los $2 \mathrm{~mm}$ y abundantes porosidades y una de las vértebras dorsales además del gran desarrollo osteofítico mostró en la cara superior una oquedad compatible con un nódulo de schmorl.

El otro esqueleto (Individuo 2) corresponde a un adulto joven representado por dos elementos óseos únicamente -un radio y un fémur. La robustez de los mismos y el diámetro de la cabeza femoral permiten proponer que se trata de un individuo masculino. No se observó pigmento sobre los huesos ni señales de osteoartritis.

Por debajo del paquete secundario fue hallado el esqueleto de un individuo articulado. El Individuo 3 estaba en posición dorsal con los miembros inferiores extendidos. Se trata de un adulto joven-adolescente (18-20 años). Las partes presentes son: el cráneo, el radio derecho, parte del brazo izquierdo, las vértebras dorsales, costillas, esternón, los coxales, el sacro, parte del cóccix y ambas piernas. Es interesante que el esqueleto presentaba abundante colorante sobre los huesos e incluso se recuperaron bochones de ocre debajo de la pelvis.

El resto de los huesos aparecieron de forma dispersa, aunque en algunos casos se observó la articulación de dos o tres elementos, sin que se pudiera reconocer la modalidad de inhumación. Pudimos determinar que los restos corresponden a un número mínimo de cuatro individuos. De éstos dos son adultos, uno es un adulto joven-adolescente y el otro es un perinato. De los dos individuos adultos (Individuos 4 y 5) uno pudo ser determinado como de sexo masculino, sin que se pudiera asignar sexo al resto de los elementos. El cálculo del MAU\% refleja que los huesos más representados son los cráneos, mandíbulas y húmeros. También se observa que casi todos los elementos se encuentran presentes a excepción del sacro y esternón. Dentro de este conjunto se recuperaron huesos con ocre sobre la superficie y otros con signos de quemado. Es interesante que en dos fémures (correspondientes a un mismo individuo) se observó una reacción perióstica que afectó a ambos elementos a nivel de la diáfisis distal y la metafisis, sin que se vea el hueso subcondral afectado. Ambos fémures muestran modificada su morfología a causa de esta reacción ósea. No se detectó la presencia de reacciones periósticas en ninguno de los otros huesos del conjunto.

Individuo 6. Se trata de los restos aislados correspondientes a un adulto joven-adolescente de 18-22 años, en el cual no se pudo establecer la modalidad de inhumación. El mismo está representado en su mayoría por huesos largos, ambos cúbitos, radio derecho, los dos peronés, las dos tibias y algunos fragmentos de costillas. El 60\% de estos huesos estaba teñido con ocre rojo.

Finalmente, el Individuo 7 es un infante (neonato). Los huesos presentes son los temporales, frontal, radio, cúbito, ambas tibias y un hueso largo indeterminado. Todos los elementos tenían abundante ocre rojo y los mismos aparecieron asociados a los restos del adulto de sexo indeterminado ya descripto.

Un caso excepcional lo constituye el Individuo 8. Se trata de los elementos óseos de un adulto quemados incluidos dentro de un fogón ubicado en la excavación central del cerrito. 
Los restos corresponden a partes del cráneo, una clavícula, una rótula, una diáfisis de hueso largo. Es interesante que más del $50 \%$ de los huesos presentara ocre sobre la superficie. Para estos restos se obtuvo un fechado de $775 \pm 85$ años AP (AA-93218). Además de los restos humanos se encontraron dentro del fogón huesos de fauna termoalterados y algunos tiestos de cerámica.

\section{Conclusiones}

Los trabajos bioarqueológicos llevados a cabo en el sitio Los Tres Cerros 1 hasta el momento permiten reconocer que en el núcleo de inhumaciones se enterraron los restos de al menos siete individuos, a esto se suma los restos de un individuo recuperado dentro de una estructura de fogón y con signos de termoalteración. Los elementos analizados corresponden a individuos de distintos sexo y grupo etario.

Las modalidades de inhumación detectadas en el sitio corresponden a un entierro secundario múltiple y a un entierro primario simple. Esta variabilidad de formas de tratamiento de los cadáveres ha sido descripta en varios sitios del Delta del Paraná (Torres 1911; Lothrop 1932; Gaspary 1950; Loponte 2007). En ningún caso se detectó ajuar asociado con los individuos, sin embargo varios de los esqueletos presentaban la superficie teñida de ocre rojo. Esto se observó en individuos inhumados de manera secundaria, primaria y en los diferentes grupos etarios. Esto indica que el uso de pigmentos fue generalizado y no se vincula con un tipo de inhumación o con alguna categoría etaria.

En cuanto a los primeros análisis paleopatológicos, algunas tendencias preliminares pueden ser delineadas. Dentro de la serie hubo una baja frecuencia de osteoartritis, en este sentido un solo individuo adulto mayor femenino mostró varias de las articulaciones dañadas y no se detectó la presencia de la patología en ningún otro individuo. Esto podría indicar que las actividades desarrolladas por el grupo no tuvieron un alto impacto sobre las articulaciones, lo cual contrasta con los datos obtenidos para grupos de cazadores-recolectores de otras áreas (Scabuzzo 2010).

Se relevaron reacciones periósticas en dos fémures correspondientes a un mismo individuo (adulto-masculino). Las características de la afección y su distribución bilateral permiten inferir la presencia de algún tipo de infección sistémica más que el resultado de un traumatismo. Sin embargo, es necesario realizar radiografías y ampliar la muestra para ver si aparecen otros elementos afectados. Finalmente, no se detectaron señales de traumatismo en los elementos, ni signos de hiperostosis porótica y criba orbitalia a nivel de los cráneos. La ausencia de hiperostosis porótica y criba orbitalia indica que no fueron comunes los eventos de estrés metabólico en los individuos (Ortner 2003). Sin embargo, para dar mayor apoyo a este punto es necesario ampliar la muestra y relevar otros indicadores tales como líneas de Harris e hipoplasia del esmalte.

Finalmente, es interesante mencionar que en el sitio Cerro Grande de la Isla de Los Marinos (Gaspary en 1950) ubicado en el departamento de Victoria en cercanías de Los Tres Cerros, se encontró un contexto semejante. Ambos sitios presentan similitudes en la variabilidad de prácticas mortuorias registradas así como en la aplicación de ocre sobre algunos de los elementos y en la presencia de huesos con signos de termoalteración. Por otro lado los dos fechados radiocarbónicos de $590 \pm 60$ y 660 70 años AP (Kozameh y Brunás 2011) son cercanos a aquellos obtenidos para los Tres Cerros. 


\section{Agradecimientos}

Nuestro agradecimiento a G. Politis y M Bonomo directores del proyecto. Al Consejo Nacional de Ciencia y Técnica (PIP-112-200801-01282) por la financiación de parte de las investigaciones. Finalmente a Jorge Suby por ayudarnos a diagnosticar parte del material.

\section{Bibliografía citada}

Bonomo, M., G. Politis y C. García Gianotti.

2011. Montículos, jerarquía social y horticultura en las sociedades indígenas del delta del Río Paraná (argentina). Latin American Antiquity 22(3): 297-333.

Buikstra, J.E. y D.H. Ubelaker.

1994. Standards for Data Collection from Human Skeletal Remains. Arkansas Archaeological Survey Research Series No 44, Arkansas.

Ceruti, C.

2003. Entidades culturales presentes en la cuenca del Paraná Medio (margen entrerriana). Mundo de Antes 3:111-135.

Gaspary, F.

1950. Investigaciones Arqueológicas y Antropológicas en un Cerrito de la Isla Los Marinos (Pcia. de Entre Ríos). Instituto de Arqueología, Lingüística y Folklore 23:3-66.

Kozameh, L. y O. Brunás

2011. Paleopatología: Paget óseo en un resto prehispánico. Microscopía y datación. Actualizaciones en Osteología 7 (2): 93-95.

Loponte, D.

2007. La economía prehistórica del norte bonaerense. Arqueología del humedal del Paraná inferior, los Bajíos Ribereños meridionales. Tesis doctoral inédita. Facultad de Ciencias Naturales y Museo, UNLP, La Plata.

Lothrop, S.

1932. Indians of the Paraná Delta, Argentina. Annals of the New York Academy of Science 32: 77-232.

Ortner D.

2003. Identification of pathological conditions in human skeletal remains. Academic Press, Nueva York.

Politis, G. M. Bonomo, C. Castiñeira y A. Blasi.

2011. Archaeology of the Upper Delta of the Paraná River (Argentina): Mound Construction and Anthropic Landscapes in the Los Tres Cerros locality. Quaternary International 245: 74-88.

Ribot, I. y C. Roberts.

1996. A Study of Non specific Stress Indicators and Skeletal Growth in Two Mediaeval Subadult Populations. Journal of Archaeological Science 23: 67-79. 
Scabuzzo, C.

2010. Actividades, patologías y nutrición de los cazadores recolectores pampeanos. Tesis Doctoral inédita, Facultad de Ciencias Naturales y Museo, UNLP.

Torres, L. M.

1911 Los primitivos habitantes del Delta del Paraná. Biblioteca Centenaria 4, Universidad Nacional de La Plata, Buenos Aires.

Ubelaker, D.

1999. Human Skeletal Remains. Taraxacum, Washington D.C.

White, T. y P. Folkens

2005. The Human Bones Manual. Elsevier Academic Press, Londres. 